\section{Microdeletions in the human H19 DMR result in loss of IGF2 imprinting and Beckwith- Wiedemann syndrome}

\author{
Angela Sparago $^{1}$, Flavia Cerrato ${ }^{1}$, Maria Vernucci ${ }^{1}$, \\ Giovanni Battista Ferrero ${ }^{2}$, Margherita Cirillo Silengo ${ }^{2}$ \\ \& Andrea Riccio ${ }^{1}$
}

The overgrowth- and tumor-associated Beckwith-Wiedemann syndrome results from dysregulation of imprinted genes on chromosome 11p15.5. Here we show that inherited microdeletions in the $\mathrm{H} 19$ differentially methylated region (DMR) that abolish two CTCF target sites cause g this disease. Maternal transmission of the deletions results in hypermethylation of the H19 DMR, biallelic IGF2 expression, H19 silencing and Beckwith-Wiedemann syndrome, indicative of loss of function of the IGF2-H19 imprinting control element.

A subclass of individuals with Beckwith-Wiedemann syndrome (BWS; OMIM 130650) show hypermethylation and silencing of the maternal $H 19$ allele and activation of both parental IGF2 alleles ${ }^{1,2}$. Upregulation of IGF2 is important for the somatic overgrowth and tumor predisposition of BWS; the role of the putative tumor-suppressor gene H19 is uncertain ${ }^{3,4}$. No mutation has yet been associated with these epigenetic alterations. In mice, a targeted deletion showed that imprinting of the paternally expressed Igf2 and maternally expressed H19 genes is controlled by a 2-kb DMR located 5' of H19 (ref. 5). The DMR contains a chromatin boundary that is methylated on the paternal chromosome and interacts with the zinc finger protein CTCF on the maternal chromosome ${ }^{6-9}$. This binding is required to maintain the unmethylated status and the insulator function of the maternal $\mathrm{DMR}^{10-12}$.

We analyzed seven individuals with BWS with $H 19$ hypermethylation for the presence of deletions in the H19 DMR. The H19 DMR sequence differs from the homologous mouse region in that it is organized into two repeat units, each consisting of two types of direct repeats, and contains seven potential binding sites for CTCF (Fig. 1a) ${ }^{13}$. We analyzed by Southern blotting a region spanning all the repeats and found an allele with a $1.8-\mathrm{kb}$ deletion in two individuals (Fig. 1b). We then amplified DNA fragments encompassing the deletion's breakpoints by PCR and sequenced them. The two mutations were very similar, each deleting 2.5 copies of the B repeat and one copy of A repeat and abolishing two CTCF sites (Fig. 1a). Both deletions were generated by recombination of the B6 repeat with the B3 repeat and differed only with respect to the cross-over breakpoints, which were $20 \mathrm{bp}$ apart. The sister of proband 1 died prenatally with signs of BWS. By PCR amplification of an 88-bp fragment encompassing the mutation from an autopsy specimen, we found that she also inherited the $1.8-\mathrm{kb}$ deletion from her mother (data not shown). We then looked for the presence of the deletion in the relatives of the probands who had no features of BWS. In family 1, we found the mutation in the mother, maternal grandfather, an uncle and his daughters (Fig. 1b). In family 2, we found the mutation in the mother, maternal aunt and maternal grandfather, indicating that the $1.8-\mathrm{kb}$ deletion is associated with the BWS phenotype only when maternally transmitted (Fig. 1b). We did not detect the $1.8-\mathrm{kb}$ deletion in any of 14 individuals with BWS with defects other than $H 19$ hypermethylation or in any of 50 healthy individuals (data not shown).

We first determined the effect of the $1.8-\mathrm{kb}$ deletion on the methylation of the H19 DMR by digestion with HpaII and Southern blotting (Fig. 2a). We observed the normal pattern of 50\% methylation, indicating differential methylation of the parental alleles, in the fathers of the probands. In their mothers, the deleted paternal allele $(\Delta \mathrm{R} 1-\mathrm{R} 4)$ was methylated whereas the wild-type maternal allele $(\mathrm{H} 2-$ R2) was not. We observed a similar methylation pattern in the other unaffected carriers (data not shown). In the probands, however, both the deleted maternal allele $(\Delta \mathrm{R} 1-\mathrm{R} 4)$ and the wild-type paternal allele (R1-R2 + R3-R4) were intensely methylated at all the HpaII sites that we analyzed.

We then determined the methylation of the DNA regions encompassing the CTCF sites in the probands and their mothers by bisulfite genomic sequencing. The repeat (B1) containing CTCF site 6 is differentially methylated on the parental alleles of normal individuals ${ }^{14}$. We observed intense hypermethylation in the probands (22 of 25 and 21 of 24 template molecules, respectively) but normal paternal-specific methylation in their mothers (Fig. $\mathbf{2 b}$ and data not shown). The repeats containing the other CTCF sites were reported to be highly methylated in normal embryos, but the methylation extent of each parental allele was not assessed ${ }^{14}$. By sequencing across the telomeric breakpoints of the 1.8 -kb deletions, we determined the allele-specific methylation of the repeat containing CTCF site 4 . In the mothers, this region normally had paternal-specific methylation (Fig. 2c and data not shown), but both the paternal CTCF site 4 (included in the B3 repeat) and the maternal CTCF site 4 (included in the hybrid B6/B3 repeat) were methylated in the probands (Fig. 2c).

We also determined the methylation of CTCF sites 2 and 3. These sequences are included in the $1.8-\mathrm{kb}$ deletions; therefore, only the maternal allele is present in the mothers and only the paternal allele in the probands. These sequences were hypomethylated in the mothers

${ }^{1}$ Dipartimento di Scienze Ambientali, Seconda Università di Napoli, via Vivaldi 43, 81100 Caserta, Italy. ${ }^{2}$ Dipartimento di Scienze Pediatriche e dell'Adolescenza, Università di Torino, 10126 Torino, Italy. Correspondence should be addressed to A.R. (andrea.riccio@unina2.it).

Published online 15 August 2004; doi:10.1038/ng1410 

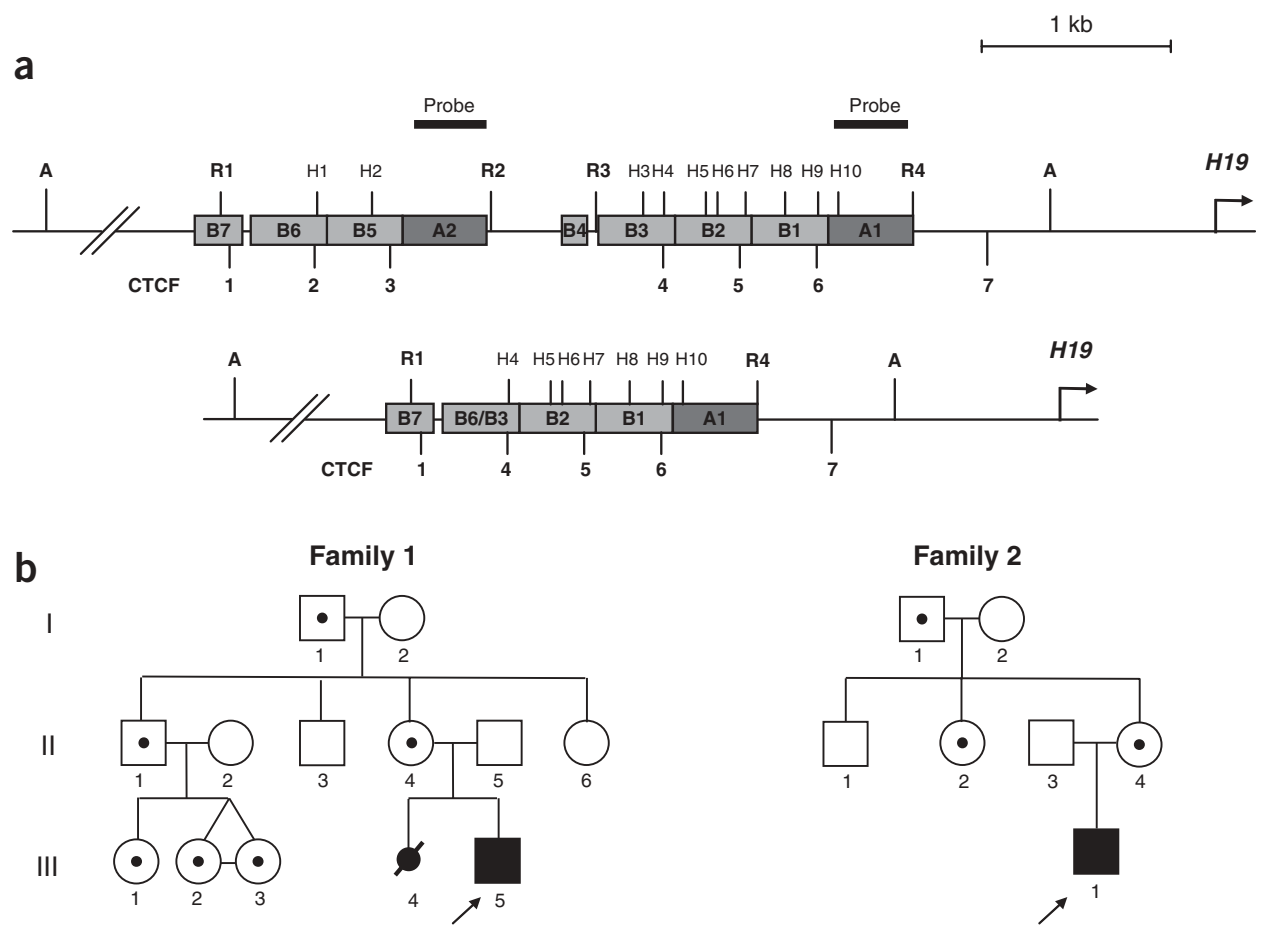

Family 2

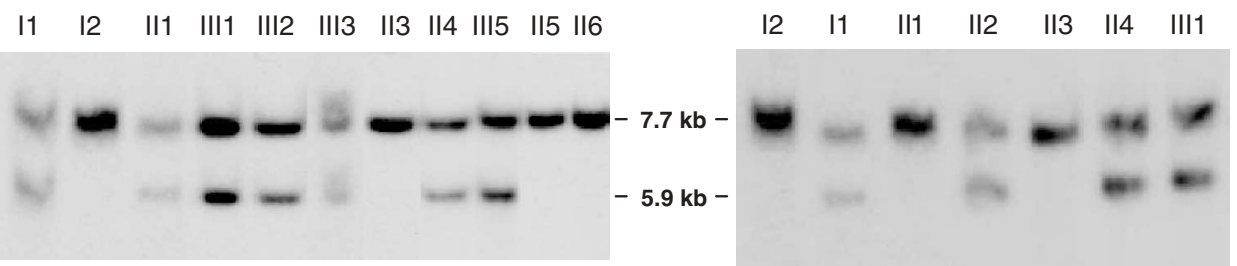

Figure 1 Identification of the 1.8-kb deletions in the H19 DMR. (a) Structure of the wild-type and mutated H19 DMR. The wild-type allele is depicted above the deleted allele. The deletion breakpoints are at -4822/-4810 and -2988/-2976 (relative to the H19 transcription start site) in family 1 and at $-4800 /-4783$ and $-2966 /-2949$ in family 2. (b) Analysis of the 1.8-kb deletions. Blood leukocyte DNAs were digested with Apal and analyzed by Southern-blot hybridization with the probe indicated in a. This probe hybridizes at two locations owing to the high homology between the A repeats. In individual III4 of family 1 , the deletion was identified by PCR amplification and DNA sequencing. Unaffected carriers are indicated by targeted symbols.

and hypermethylated in the probands (Fig. 2d and data not shown), indicating that CTCF sites 2 and 3 are also normally preferentially methylated on the paternal allele. Thus, the 1.8 -kb deletion abolishes two paternally methylated CTCF sites and its maternal transmission results in hypermethylation of the remaining part of the H19 DMR.

Next, we examined the effect of the $1.8-\mathrm{kb}$ deletion on the expression of IGF2 and H19. We isolated RNA from fibroblasts derived from skin biopsies of proband 1 and his mother and from primary cells derived from a tongue biopsy of proband 2. Amplification of transcribed polymorphisms by RT-PCR allowed us to determine the allele-specific expression of IGF2. Both alleles were expressed in skin fibroblasts from proband 1 (maternal:paternal ratio 1:2) and in tongue cells from proband 2 (maternal:paternal ratio 1:1), but one allele was mainly expressed in the mother of proband 1 (maternal: paternal ratio 1:10; Fig. 2e). We assessed the total level of H19 RNA in the skin fibroblasts by quantitative real-time RT-PCR (Fig. 2f). H19 expression in proband 1 was 34 times lower than in his mother, showing that loss of IGF2 imprinting was accompanied by downregulation of $H 19$.

By showing that internal deletions of the human H19 DMR affect the imprinted expression of IGF2 and H19, we define the imprinting control element of the locus and indicate how its mutation can result in BWS. The activation of the maternal IGF2 allele and the suppression of $H 19$ are probably caused by loss of the insulator function due to hypermethylation of the maternal DMR (Supplementary Fig. 1 online). This can be the result of inefficient protection from de novo methylation in somatic cells or lack of erasure of the grandpaternal imprint in the maternal germ line.

Most cases of BWS occur sporadically and are believed to result from 'pure' epigenetic errors ${ }^{1,2}$. Our results indicate that at least some of the DNA-methylation defects are caused by mutation in an imprinting regulatory region. Similar imprinting mutations have been found on chromosome 15q11-q13 in individuals affected by Prader-Willi and Angelman syndromes ${ }^{15}$. Such mutations can be transmitted silently through the germ line of one sex, but result in imprinting defects when they go through the germ line of the opposite sex.

All the genetic analyses were done after informed consent was obtained from the parents of the affected individuals. The experimental plan was approved by the ethical committee of the Second University of Naples, Italy (Supplementary Methods online).

GenBank accession number. H19, AF125183. 
a

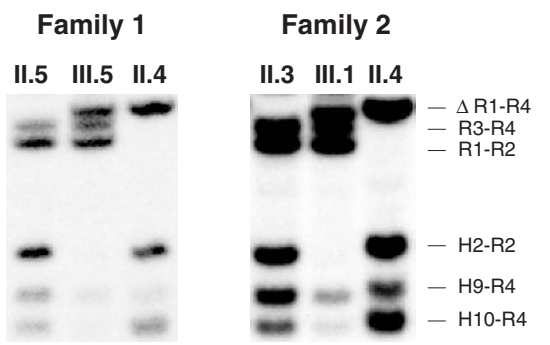

b

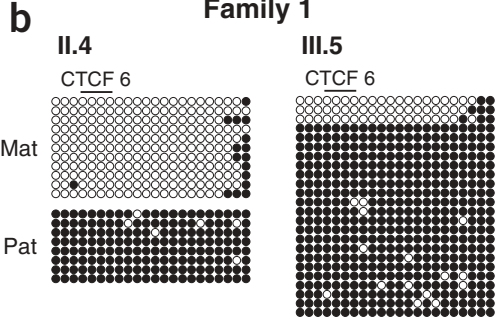

Family 2

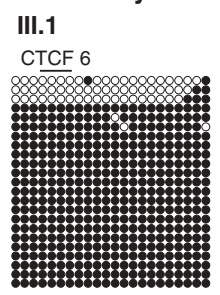

c

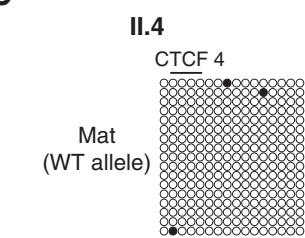

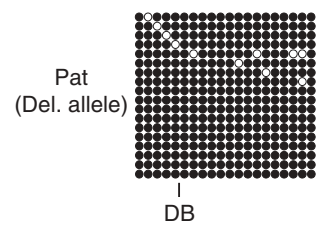

e

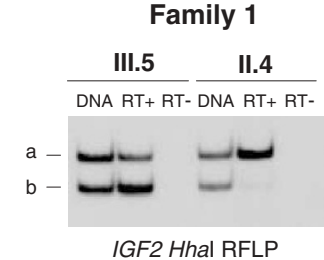

Family 1

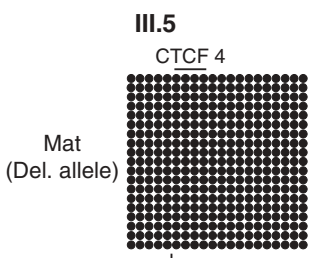

$\stackrel{1}{\mathrm{DB}}$

Pat
(WT allele)
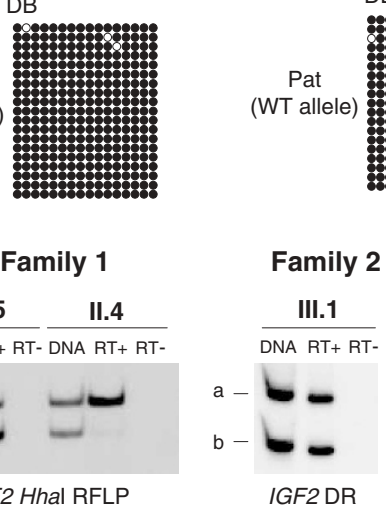

Family 2

III.1

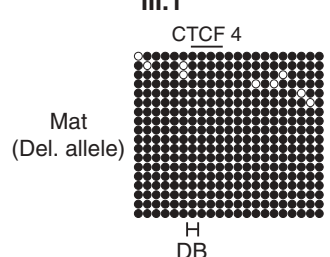

Pat

(WT allele) d

II.4

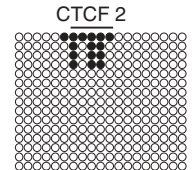

III.5

CTCF 2

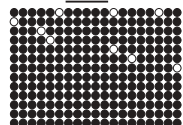

Family 1

II.4

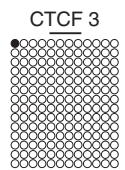

III.5

CTCF 3

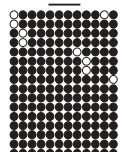

Figure 2 Effect of the 1.8-kb deletion on imprinted methylation and expression of the IGF2-H19 locus. (a) DNA methylation of the H19 DMR determined by Southern blotting. Blood leukocyte DNAs of the probands (III5 and IIII) and their parents (II4 and II5, and II4 and II3) in families 1 and 2, respectively, were digested with Hpall and Rsal and hybridized with the probe indicated in Figure 1a. (b-d) DNA methylation profiles of CTCF site 6 (b), CTCF site 4 (c) and CTCF sites 2 and 3 (d) and flanking regions determined by bisulfite genomic sequencing. The results from the probands and one of the mothers are shown. Each line corresponds to a single template DNA molecule cloned; each circle corresponds to a CpG dinucleotide. Filled circles designate methylated cytosines; open circles, unmethylated cytosines. The CpGs included in the CTCF sites are indicated. The maternal (Mat) and paternal (Pat) alleles of the CTCF site 6 region of the mother were discriminated by known single-nucleotide polymorphisms. The bisulfite sequencing of CTCF site 4 crossed the telomeric breakpoints of the 1.8-kb deletions (DB) and different forward primers were used for the deleted (Del) and wild-type (WT) alleles. (e) Allele-specific expression of IGF2. RNAs extracted from cultured skin fibroblasts of proband 1 (III5) and his mother (II4) in family 1 and from tongue tissue of proband 2 (III1) in family 2 were analyzed by hot-stop RT-PCR. The parental alleles were discriminated by typing for a Hhal restriction fragment length polymorphism (RFLP) in family 1 and by typing for a direct repeat (DR) in family 2. As control, the RNAs were amplified with (RT +) and without (RT-) previous treatment with reverse transcriptase. (f) Determination of the H19 RNA level. RNAs derived from the fibroblasts of proband 1 and his mother in family 1 were analyzed by quantitative real-time RT-PCR using primers specific for the $\mathrm{H} 19$ transcript and the GAPD mRNA.

Note: Supplementary information is available on the Nature Genetics website.

\section{ACKNOWLEDGMENTS}

We thank P. Carotenuto and M. Zollo for help in the use of the real-time PCR, W. Reik and M. Constancia for critical reading of the manuscript, M.V. Cubellis for support and the affected individuals and their families for their collaboration. This work was supported by grants from the fund Progetti di Ricerca di Interesse Nazionale 2003 of the Italian Ministero dell'Istruzione, dell'Università e della Ricerca (to A.R.) and from the Compagnia di San Paolo di Torino (to G.B.F.).

\section{COMPETING INTERESTS STATEMENT}

The authors declare that they have no competing financial interests.

Received 7 May; accepted 14 July 2004

Published online at http://www.nature.com/naturegenetics/

1. Maher, E.R. \& Reik, W. J. Clin. Invest. 105, 247-252 (2000).
2. Weksberg, R., Smith, A.C., Squire, J. \& Sadowski, P. Hum. Mol. Genet. 12, R61-R68 (2003).

3. Eggenschwiler, J. et al. Genes. Dev. 11, 3128-3142 (1997).

4. Feinberg, A.P., Cui, H. \& Ohlsson, R. Semin. Cancer Biol. 12, 389-398 (2002).

5. Thorvaldsen, J.L., Duran, K.L. \& Bartolomei, M.S. Genes. Dev. 12, 3693-3702 (1998).

6. Hark, A.T. et al. Nature 405, 486-489 (2000).

7. Bell, A.C. \& Felsenfeld, G. Nature 405, 482-485 (2000).

8. Kanduri, C. et al. Curr. Biol. 10, 853-856 (2000).

9. Szabo, P., Tang, S.H., Rentsendorj, A., Pfeifer, G.P. \& Mann, J.R. Curr. Biol. 10, 607-610 (2000).

10. Schoenherr, C.J., Levorse, J.M. \& Tilghman, S.M. Nat. Genet. 33, 66-69 (2003).

11. Pant, V. et al. Genes. Dev. 17, 586-590 (2003).

12. Szabo, P.E., Tang, S.H., Silva, F.J., Tsark, W.M. \& Mann, J.R. Mol. Cell. Biol. 24, 4791-4800 (2004).

13. Frevel, M.A., Sowerby, S.J., Petersen, G.B. \& Reeve, A.E. J. Biol. Chem. 274, 29331-29340 (1999).

14. Takai, D., Gonzales, F.A., Tsai, Y.C., Thayer, M.J. \& Jones, P.A. Hum. Mol. Genet. 10, 2619-2626 (2001).

15. Buiting, K. et al. Nat. Genet. 9, 395-400 (1995). 